\title{
Correction to: Efficacy and Safety of Supportive Care Biosimilars Among Cancer Patients: A Systematic Review and Meta-Analysis
}

\author{
Jichun Yang ${ }^{1}$. Shuqing $\mathrm{Yu}^{1} \cdot$ Zhirong Yang $^{2} \cdot$ Yusong Yan ${ }^{1} \cdot$ Yao Chen ${ }^{1} \cdot$ Hongmei Zeng $^{3} \cdot$ Fei Ma ${ }^{4} \cdot$ Yanxia Shi $^{5}$. \\ Yehui Shi ${ }^{6} \cdot$ Zilu Zhang $^{7} \cdot$ Feng Sun ${ }^{1}$ (I)
}

Published online: 21 September 2019

(c) Springer Nature Switzerland AG 2019

\section{Correction to: BioDrugs (2019) 33:373-389 https://doi.org/10.1007/s40259-019-00356-3}

The authors unintentionally included in the meta-analysis both the initial abstract and the final paper of the study by Puertolas et al. [45, 48]. In order to remove this duplication, the following corrections are required.

Page 373, abstract, results, line 1: The following sentence, which previously read:

The original article can be found online at https://doi.org/10.1007/ s40259-019-00356-3.

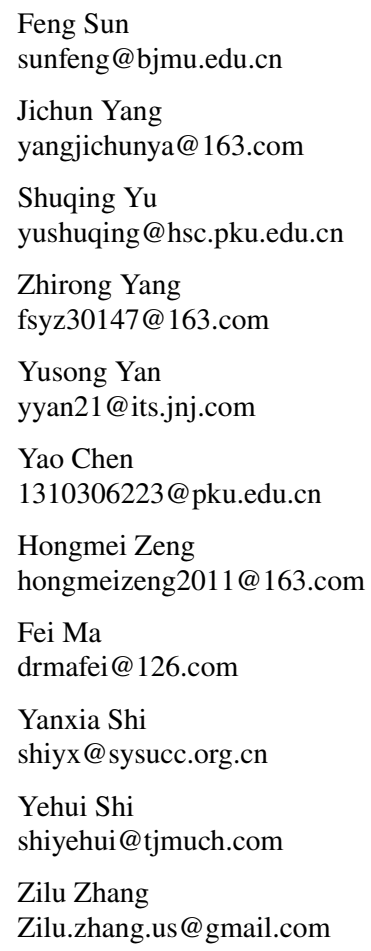

"We identified 29 studies that compared biosimilars of G-CSF or epoetin alfa: one RCT and five cohort studies (total $\mathrm{N}=2816$ ) of epoetin alfa biosimilars, and 13 RCTs and 10 cohort studies (total $\mathrm{N}=23,561$ ) of G-CSF biosimilars."

should read:

"We identified 28 studies that compared biosimilars of G-CSF or epoetin alfa: one RCT and five cohort studies (total $\mathrm{N}=2816$ ) of epoetin alfa biosimilars, and $13 \mathrm{RCTs}$ and 9 cohort studies (total $\mathrm{N}=23,043$ ) of G-CSF biosimilars."

1 Department of Epidemiology and Biostatistics, School of Public Health, Peking University, 38 Xueyuan Road, Haidian District, Beijing 100191, China

2 Primary Care Unit, School of Clinical Medicine, University of Cambridge, Cambridgeshire CB1 8RN, UK

3 Department of Cancer Registry, National Cancer Center/National Clinical Research Center for Cancer/Cancer Hospital, Chinese Academy of Medical Sciences and Peking Union Medical College, Beijing 100021, China

4 Department of Medical Oncology, National Cancer Center/National Clinical Research Center for Cancer/Cancer Hospital, Chinese Academy of Medical Sciences and Peking Union Medical College, Beijing 100021, China

5 Department of Medical Oncology, Sun Yat-Sen University Cancer Center/State Key Laboratory of Oncology in South China/Collaborative Innovation Center for Cancer Medicine, Guangzhou 510060, China

6 Phase I Clinical Trial Department of Tianjin Medical University Cancer Institute and Hospital, Tianjin 300060, China

7 Harvard Medical School and Harvard Pilgrim Health Care Institute, Boston, MA 02215, USA 
Page 377, fig. 1, the following text, which previously read:

Box 8: "Full-text excluded $(\mathrm{n}=211)$ " should read "Fulltext excluded $(\mathrm{n}=212) "$

"Duplicates: 91" should read "Duplicates 92"

Box 9: "Full-text articles assessed for eligibility $(n=29)$ " should read "Full-text articles assessed for eligibility $(\mathrm{n}=28)$ "

Box 10: "Studies included in qualitative synthesis $(\mathrm{n}=29)$ " should read "Studies included in qualitative synthesis $(\mathrm{n}=28)$ "

Box 11: 'Studies included in quantitative synthesis $(\mathrm{n}=29)$ " should read "Studies included in quantitative synthesis $(\mathrm{n}=28)$ "

"G-CSF biosimilars:13 RCTs + 10 Cohort studies" should read "G-CSF biosimilars:13 RCTs + 9 Cohort studies"

A corrected version of Fig. 1 is shown below:
Page 379, Table 1, 'G-CSF biosimilars vs. G-CSF' section, 'Puertolas et al. (2016) [45]' row: the entire row should be deleted.

Page 379, Table 1, 'G-CSF biosimilars vs. G-CSF' section, 'Puertolas et al. (2018) [48]' row: The cell entry in column 4 'Sample size (B/R)', which previously read "303/215" should read "49/49".

Page 380, Table 1, 'G-CSF biosimilars vs. G-CSF' section, 'Total patients of cohort studies' row: the cell entry in column 4 'Sample size (B/R)', which previously read “2677/17,739" should read "2374/17,524".

Page 381, section 3.2.2, paragraph 1, line 1: The sentence, which previously read: "Ten cohort studies (2677 patients vs 17,739 patients)..." should read "Nine cohort studies (2374 patients vs 17,524 patients)...”.

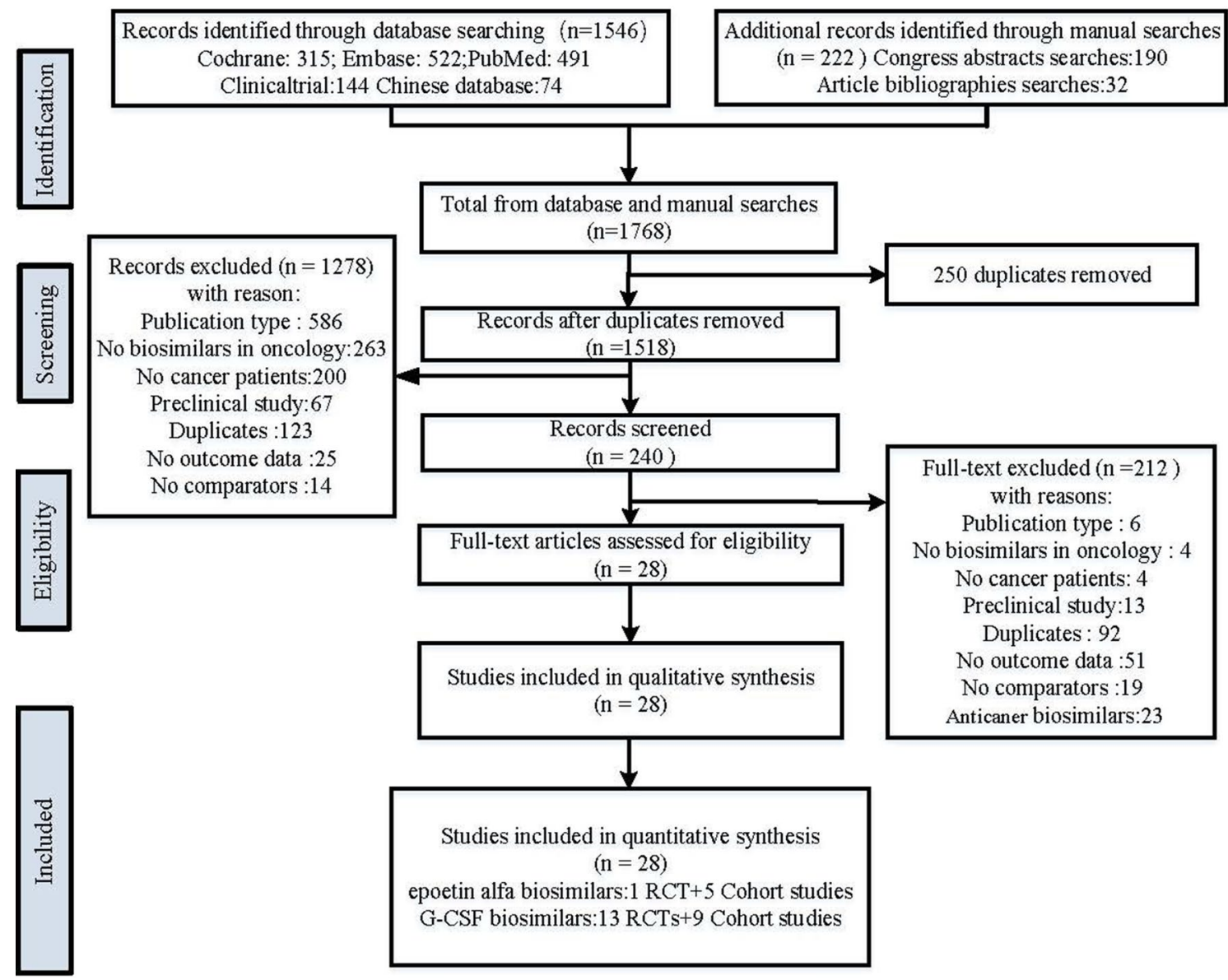

Fig. 1 PRISMA flowchart of included studies. G-CSF granulocyte colony-stimulating factors, $R C T$ randomized controlled trial 
Page 381, section 3.2.2, paragraph 3, line 10: The sentence, which previously read

"In addition, five cohort studies [40, 41, 45, 48, 49] compared the incidence of $\mathrm{FN}$ in cycle 1 in cancer patients treated with filgrastim biosimilars (860 patients) and filgrastim (6970 patients)."

should read

"In addition, four cohort studies [40, 41, 48, 49] compared the incidence of $\mathrm{FN}$ in cycle 1 in cancer patients treated with filgrastim biosimilars (557 patients) and filgrastim (6755 patients)."

Page 382, Table 3, 'FN incidence in cycle 1 ( 3 wk)' section, 'Cohort study' study type: the values in the 'Cancer type/Breast cancer' row and the 'Total' row have been corrected. A corrected version of the table is shown below with the corrected text shown in bold.

Table 3 Results for G-CSF biosimilars

\begin{tabular}{|c|c|c|c|c|c|c|c|c|c|c|c|c|}
\hline \multirow{2}{*}{$\begin{array}{l}\text { Outcomes } \\
\text { (follow-up } \\
\text { time) }\end{array}$} & \multirow[t]{2}{*}{$\begin{array}{l}\text { Study } \\
\text { type }\end{array}$} & \multirow{2}{*}{$\begin{array}{l}\text { Group } \\
\text { factors }\end{array}$} & \multirow[t]{2}{*}{ Subgroup } & \multirow{2}{*}{$\begin{array}{l}\text { No. } \\
\text { study }\end{array}$} & \multirow{2}{*}{$\begin{array}{l}\text { Sample size } \\
(\mathrm{B} / \mathrm{R})\end{array}$} & \multicolumn{2}{|c|}{$\begin{array}{l}\text { Heterogeneity } \\
\text { test }\end{array}$} & \multicolumn{3}{|c|}{ Results of meta-analysis } & \multirow{2}{*}{$\begin{array}{l}p \text {-Value } \\
\text { between } \\
\text { sub- } \\
\text { groups }\end{array}$} & \multirow{2}{*}{$\begin{array}{l}\text { GRADE } \\
\text { evidence }\end{array}$} \\
\hline & & & & & & $\overline{I^{2}}$ & $\begin{array}{l}p \\
\text { value }\end{array}$ & $\begin{array}{l}\text { Summary } \\
\text { effects }\end{array}$ & $95 \% \mathrm{CI}$ & $\begin{array}{l}p \\
\text { value }\end{array}$ & & \\
\hline \multirow[t]{10}{*}{$\begin{array}{l}\text { FN inci- } \\
\text { dence in } \\
\text { cycle } 1 \\
(3 \mathrm{wk})\end{array}$} & \multirow[t]{6}{*}{ RCT } & \multirow[t]{2}{*}{$\begin{array}{l}\text { Drug } \\
\text { type }\end{array}$} & $\begin{array}{l}\text { Fil- } \\
\text { grastim } \\
\text { biosimi- } \\
\text { lars }\end{array}$ & $\begin{array}{l}4[53, \\
56-58]\end{array}$ & $470 / 352$ & $10.8 \%$ & 0.339 & $\mathrm{RR}=1.09$ & $\begin{array}{c}0.72 \text { to } \\
1.65\end{array}$ & 0.19 & 0.22 & \multirow[t]{6}{*}{ Low } \\
\hline & & & $\begin{array}{l}\text { Pegfil- } \\
\text { grastim } \\
\text { biosimi- } \\
\text { lars }\end{array}$ & $\begin{array}{l}4[28, \\
50,51, \\
59]\end{array}$ & $582 / 515$ & $0.0 \%$ & 0.81 & $\mathrm{RR}=1.14$ & $\begin{array}{c}0.73 \text { to } \\
1.79\end{array}$ & 0.57 & & \\
\hline & & \multirow[t]{3}{*}{$\begin{array}{c}\text { Cancer } \\
\text { type }\end{array}$} & $\begin{array}{l}\text { Breast } \\
\text { cancer }\end{array}$ & $\begin{array}{l}5[28, \\
50,56, \\
57,59]\end{array}$ & $738 / 713$ & $0.0 \%$ & 0.90 & $\mathrm{RR}=1.14$ & $\begin{array}{c}0.80 \text { to } \\
1.63\end{array}$ & 0.47 & \multirow[t]{4}{*}{0.22} & \\
\hline & & & NSCLC & $\begin{array}{c}2[51, \\
53]\end{array}$ & $251 / 125$ & $0.0 \%$ & 0.64 & $\mathrm{RR}=1.53$ & $\begin{array}{c}0.80 \text { to } \\
2.93\end{array}$ & 0.19 & & \\
\hline & & & NHL & $1[58]$ & $63 / 29$ & & & $\mathrm{RR}=0.54$ & $\begin{array}{c}0.20 \text { to } \\
1.46\end{array}$ & 0.22 & & \\
\hline & & Total & $\begin{array}{l}\text { G-CSF } \\
\text { biosimi- } \\
\text { lars }\end{array}$ & $\begin{array}{l}8 \text { [28, } \\
50,51, \\
53, \\
55-59]\end{array}$ & $1052 / 867$ & $0.0 \%$ & 0.74 & $\mathrm{RR}=1.09$ & $\begin{array}{c}0.80 \text { to } \\
1.49\end{array}$ & 0.58 & & \\
\hline & \multirow[t]{4}{*}{$\begin{array}{r}\text { Cohort } \\
\text { study }\end{array}$} & \multirow[t]{3}{*}{$\begin{array}{l}\text { Cancer } \\
\text { type }\end{array}$} & $\begin{array}{l}\text { Breast } \\
\text { cancer }\end{array}$ & $\begin{array}{c}2[40, \\
48]\end{array}$ & $196 / 183$ & $\mathbf{0 . 0 \%}$ & 0.28 & $R R=1.60$ & $\begin{array}{c}0.85 \text { to } \\
3.01\end{array}$ & 0.15 & \multirow[t]{4}{*}{0.43} & \multirow[t]{4}{*}{$\begin{array}{l}\text { Moder- } \\
\text { ate }\end{array}$} \\
\hline & & & NHL & $1[41]$ & $12 / 26$ & & & $\mathrm{RR}=0.87$ & $\begin{array}{c}0.20 \text { to } \\
3.85\end{array}$ & 0.85 & & \\
\hline & & & $\begin{array}{l}\text { Non- } \\
\text { myeloid } \\
\text { cancer }\end{array}$ & $1[49]$ & $349 / 6546$ & & & $\mathrm{RR}=0.97$ & $\begin{array}{c}0.46 \text { to } \\
2.05\end{array}$ & 0.93 & & \\
\hline & & Total & $\begin{array}{l}\text { Fil- } \\
\text { grastim } \\
\text { biosimi- } \\
\text { lars }\end{array}$ & $\begin{array}{c}4[40, \\
41,48, \\
49]\end{array}$ & $557 / 6755$ & $\mathbf{0 . 0 \%}$ & 0.35 & $\mathbf{R} R=1.25$ & $\begin{array}{c}0.79 \text { to } \\
1.98\end{array}$ & 0.35 & & \\
\hline
\end{tabular}




\begin{tabular}{|c|c|c|c|c|c|c|c|c|c|c|c|c|}
\hline \multirow{2}{*}{$\begin{array}{l}\text { Outcomes } \\
\text { (follow-up } \\
\text { time) }\end{array}$} & \multirow{2}{*}{$\begin{array}{l}\text { Study } \\
\text { type }\end{array}$} & \multirow{2}{*}{$\begin{array}{l}\text { Group } \\
\text { factors }\end{array}$} & \multirow[t]{2}{*}{ Subgroup } & \multirow{2}{*}{$\begin{array}{l}\text { No. } \\
\text { study }\end{array}$} & \multirow{2}{*}{$\begin{array}{l}\text { Sample size } \\
(\mathrm{B} / \mathrm{R})\end{array}$} & \multicolumn{2}{|c|}{$\begin{array}{l}\text { Heterogeneity } \\
\text { test }\end{array}$} & \multicolumn{3}{|c|}{ Results of meta-analysis } & \multirow{2}{*}{$\begin{array}{l}p \text {-Value } \\
\text { between } \\
\text { sub- } \\
\text { groups }\end{array}$} & \multirow{2}{*}{$\begin{array}{l}\text { GRADE } \\
\text { evidence }\end{array}$} \\
\hline & & & & & & $\overline{I^{2}}$ & $\begin{array}{l}p \\
\text { value }\end{array}$ & $\begin{array}{l}\text { Summary } \\
\text { effects }\end{array}$ & $95 \% \mathrm{CI}$ & $\begin{array}{l}p \\
\text { value }\end{array}$ & & \\
\hline \multirow[t]{5}{*}{$\begin{array}{l}\text { DSN in } \\
\text { cycle } 1 \\
(3 \mathrm{wk})\end{array}$} & \multirow[t]{5}{*}{$\mathrm{RCT}$} & \multirow[t]{2}{*}{$\begin{array}{l}\text { Cancer } \\
\text { type }\end{array}$} & $\begin{array}{l}\text { Breast } \\
\text { cancer }\end{array}$ & $\begin{array}{c}7[28, \\
50,52, \\
54,57, \\
59,60]\end{array}$ & $1092 / 975$ & $0.0 \%$ & 0.70 & $\mathrm{WMD}=0.03$ & $\begin{array}{c}-0.07 \\
\text { to } \\
0.13\end{array}$ & 0.50 & 0.27 & \multirow[t]{2}{*}{$\begin{array}{l}\text { Moder- } \\
\text { ate }\end{array}$} \\
\hline & & & NHL & $1[58]$ & $63 / 29$ & & & $\mathrm{WMD}=-0.40$ & $\begin{array}{l}-1.17 \\
\text { to } \\
0.37\end{array}$ & 0.31 & & \\
\hline & & \multirow[t]{2}{*}{$\begin{array}{l}\text { Drug } \\
\text { type }\end{array}$} & $\begin{array}{l}\text { Fil- } \\
\text { grastim } \\
\text { biosimi- } \\
\text { lars }\end{array}$ & $\begin{array}{l}3[54, \\
57,58]\end{array}$ & $386 / 260$ & $0.0 \%$ & 0.37 & $\mathrm{WMD}=0.06$ & $\begin{array}{c}-0.12 \\
\text { to } \\
0.23\end{array}$ & 0.53 & 0.70 & \multirow[t]{3}{*}{$\begin{array}{l}\text { Moder- } \\
\text { ate }\end{array}$} \\
\hline & & & $\begin{array}{l}\text { Pegfil- } \\
\text { grastim } \\
\text { biosimi- } \\
\text { lars }\end{array}$ & $\begin{array}{l}5[28, \\
50,52, \\
59,60]\end{array}$ & $769 / 744$ & $0.0 \%$ & 0.58 & $\mathrm{WMD}=0.01$ & $\begin{array}{l}-0.11 \\
\text { to } \\
0.13\end{array}$ & 0.83 & & \\
\hline & & Total & $\begin{array}{l}\text { G-CSF } \\
\quad \text { biosimi- } \\
\text { lars }\end{array}$ & $\begin{array}{l}8[28, \\
50,52, \\
54, \\
57-60]\end{array}$ & $1155 / 1004$ & $0.0 \%$ & 0.66 & $\mathrm{WMD}=0.03$ & $\begin{array}{c}-0.07 \\
\text { to } \\
0.13\end{array}$ & 0.59 & & \\
\hline \multirow{4}{*}{$\begin{array}{l}\text { Time to } \\
\text { ANC } \\
\text { recovery } \\
\text { in cycle } 1 \\
\text { ( } 3 \mathrm{wk})\end{array}$} & \multirow[t]{3}{*}{ RCT } & \multirow[t]{2}{*}{$\begin{array}{c}\text { Cancer } \\
\text { type }\end{array}$} & $\begin{array}{l}\text { Breast } \\
\text { cancer }\end{array}$ & $\begin{array}{l}4[50, \\
52,59, \\
60]\end{array}$ & $587 / 569$ & $28.7 \%$ & 0.24 & $\mathrm{WMD}=0.07$ & $\begin{array}{l}-0.10 \\
\text { to } \\
0.24\end{array}$ & 0.42 & 0.84 & \multirow[t]{3}{*}{$\begin{array}{l}\text { Moder- } \\
\text { ate }\end{array}$} \\
\hline & & & NSCLC & $1[51]$ & $93 / 46$ & & & $\mathrm{WMD}=-0.07$ & $\begin{array}{c}-1.41 \\
\text { to } \\
1.27\end{array}$ & 0.92 & & \\
\hline & & Total & $\begin{array}{l}\text { Pegfil- } \\
\text { grastim } \\
\text { biosimi- } \\
\text { lars }\end{array}$ & $\begin{array}{l}5 \\
\quad[49-52 \\
59,60]\end{array}$ & $680 / 615$ & $5.8 \%$ & 0.37 & $\mathrm{WMD}=0.07$ & $\begin{array}{l}-0.10 \\
\text { to } \\
0.24\end{array}$ & 0.43 & & \\
\hline & $\begin{array}{r}\text { Cohort } \\
\text { study }\end{array}$ & NHL & $\begin{array}{l}\text { Fil- } \\
\text { grastim } \\
\text { biosimi- } \\
\text { lars }\end{array}$ & $1[41]$ & $12 / 26$ & & & $\mathrm{WMD}=-0.14$ & $\begin{array}{c}-0.42 \\
\text { to } \\
0.70\end{array}$ & 0.63 & & Low \\
\hline \multirow{5}{*}{$\begin{array}{l}\text { Bone pain } \\
\text { rate } \\
(3-30 \mathrm{wk})\end{array}$} & \multirow[t]{4}{*}{ RCT } & \multirow[t]{3}{*}{$\begin{array}{l}\text { Cancer } \\
\text { type }\end{array}$} & $\begin{array}{l}\text { Breast } \\
\text { cancer }\end{array}$ & $\begin{array}{c}2[54, \\
59]\end{array}$ & $512 / 355$ & $80.9 \%$ & 0.02 & $\mathrm{RR}=0.89$ & $\begin{array}{c}0.76 \text { to } \\
1.03\end{array}$ & 0.12 & 0.63 & \multirow[t]{4}{*}{$\begin{array}{l}\text { Moder- } \\
\text { ate }\end{array}$} \\
\hline & & & NSCLC & $1[53]$ & $158 / 79$ & & & $\mathrm{RR}=1.20$ & $\begin{array}{c}0.44 \text { to } \\
3.29\end{array}$ & 0.72 & & \\
\hline & & & $\begin{array}{l}\text { Various } \\
\text { tumors }\end{array}$ & $1[55]$ & $54 / 54$ & & & $\mathrm{RR}=1.25$ & $\begin{array}{c}0.53 \text { to } \\
2.92\end{array}$ & 0.61 & & \\
\hline & & Total & $\begin{array}{l}\text { Fil- } \\
\text { grastim } \\
\text { biosimi- } \\
\text { lars }\end{array}$ & $\begin{array}{l}4 \\
{[53-55,} \\
59]\end{array}$ & $724 / 488$ & $51.3 \%$ & 0.10 & $\mathrm{RR}=0.90$ & $\begin{array}{c}0.78 \text { to } \\
1.05\end{array}$ & 0.18 & & \\
\hline & $\begin{array}{r}\text { Cohort } \\
\text { study }\end{array}$ & $\begin{array}{l}\text { Various } \\
\text { tumors }\end{array}$ & $\begin{array}{l}\text { Fil- } \\
\text { grastim } \\
\text { biosimi- } \\
\text { lars }\end{array}$ & $\begin{array}{l}4 \\
{[42-44,} \\
46]\end{array}$ & $123 / 309$ & $0.0 \%$ & 0.61 & $\mathrm{RR}=0.86$ & $\begin{array}{c}0.59 \text { to } \\
1.24\end{array}$ & 0.41 & & $\begin{array}{l}\text { Moder- } \\
\text { ate }\end{array}$ \\
\hline
\end{tabular}




\begin{tabular}{|c|c|c|c|c|c|c|c|c|c|c|c|c|}
\hline \multirow{2}{*}{$\begin{array}{l}\text { Outcomes } \\
\text { (follow-up } \\
\text { time) }\end{array}$} & \multirow[t]{2}{*}{$\begin{array}{l}\text { Study } \\
\text { type }\end{array}$} & \multirow[t]{2}{*}{$\begin{array}{l}\text { Group } \\
\text { factors }\end{array}$} & \multirow[t]{2}{*}{ Subgroup } & \multirow[t]{2}{*}{$\begin{array}{l}\text { No. } \\
\text { study }\end{array}$} & \multirow[t]{2}{*}{$\begin{array}{l}\text { Sample size } \\
(\mathrm{B} / \mathrm{R})\end{array}$} & \multicolumn{2}{|c|}{$\begin{array}{l}\text { Heterogeneity } \\
\text { test }\end{array}$} & \multicolumn{3}{|c|}{ Results of meta-analysis } & \multirow{2}{*}{$\begin{array}{l}p \text {-Value } \\
\text { between } \\
\text { sub- } \\
\text { groups }\end{array}$} & \multirow[t]{2}{*}{$\begin{array}{l}\text { GRADE } \\
\text { evidence }\end{array}$} \\
\hline & & & & & & $\overline{I^{2}}$ & $\begin{array}{l}p \\
\text { value }\end{array}$ & $\begin{array}{l}\text { Summary } \\
\text { effects }\end{array}$ & $95 \% \mathrm{CI}$ & $\begin{array}{l}p \\
\text { value }\end{array}$ & & \\
\hline \multirow[t]{6}{*}{$\begin{array}{l}\text { ADE rate } \\
(3-30 \mathrm{wk})\end{array}$} & \multirow[t]{5}{*}{$\mathrm{RCT}$} & \multirow[t]{2}{*}{$\begin{array}{l}\text { Drug } \\
\text { type }\end{array}$} & $\begin{array}{l}\text { Fil- } \\
\text { grastim } \\
\text { biosimi- } \\
\text { lars }\end{array}$ & $\begin{array}{l}4[29, \\
54,56, \\
60]\end{array}$ & $674 / 412$ & $6.3 \%$ & 0.36 & $\mathrm{RR}=1.03$ & $\begin{array}{c}0.97 \text { to } \\
1.09\end{array}$ & 0.35 & & \multirow[t]{5}{*}{$\begin{array}{l}\text { Moder- } \\
\text { ate }\end{array}$} \\
\hline & & & $\begin{array}{l}\text { Pegfil- } \\
\text { grastim } \\
\text { biosimi- } \\
\text { lars }\end{array}$ & $\begin{array}{c}3[29, \\
51,59]\end{array}$ & $579 / 463$ & $61.8 \%$ & 0.07 & $\mathrm{RR}=0.98$ & $\begin{array}{c}0.95 \text { to } \\
1.01\end{array}$ & 0.24 & 0.16 & \\
\hline & & \multirow[t]{2}{*}{$\begin{array}{c}\text { Cancer } \\
\text { type }\end{array}$} & $\begin{array}{l}\text { Breast } \\
\text { cancer }\end{array}$ & $\begin{array}{l}6[28, \\
29,54, \\
56,59, \\
60]\end{array}$ & $1158 / 825$ & $32.8 \%$ & 0.19 & $\mathrm{RR}=0.99$ & $\begin{array}{c}0.96 \text { to } \\
1.02\end{array}$ & 0.61 & \multirow[t]{3}{*}{0.08} & \\
\hline & & & NSCLC & $1[51]$ & $95 / 50$ & & & $\mathrm{RR}=0.92$ & $\begin{array}{c}0.50 \text { to } \\
1.71\end{array}$ & 0.40 & & \\
\hline & & Total & $\begin{array}{l}\text { G-CSF } \\
\text { biosimi- } \\
\text { lars }\end{array}$ & $\begin{array}{l}7[28, \\
29,51, \\
54,56, \\
59,60]\end{array}$ & $1253 / 875$ & $42.4 \%$ & 0.10 & $\mathrm{RR}=0.98$ & $\begin{array}{c}0.95 \text { to } \\
1.02\end{array}$ & 0.39 & & \\
\hline & $\begin{array}{r}\text { Cohort } \\
\text { study }\end{array}$ & $\begin{array}{l}\text { Various } \\
\text { tumors }\end{array}$ & $\begin{array}{l}\text { Fil- } \\
\text { grastim } \\
\text { biosimi- } \\
\text { lars }\end{array}$ & $1[47]$ & $1694 / 10,460$ & & & $\mathrm{RR}=1.08$ & $\begin{array}{c}0.89 \text { to } \\
1.31\end{array}$ & 0.43 & & $\begin{array}{l}\text { Moder- } \\
\text { ate }\end{array}$ \\
\hline
\end{tabular}

$A D E$ adverse drug event, $A N C$ absolute neutrophil count, $B / R$ biosimilars/reference biologics, $C I$ confidence interval, $D S N$ duration of severe (grade 4) neutropenia, $F N$ febrile neutropenia, G-CSF granulocyte colony-stimulating factors, GRADE Grading of Recommendations Assessment, Development and Evaluation, NHL non-Hodgkin's lymphoma, No. study number of included studies, NSCLC nonsquamous non-smallcell lung cancer, $R C T$ randomized controlled trial, $R R$ risk ratio, $W M D$ weighted mean difference

\section{Page 384, Fig. 3, 'FN incidence in cycle1.cohort study' section, 'Breast cancer' subgroup:}

- the text in the 'Study number' column that previously read " 3 " should read " 2 ";

- the text in the 'Biosimilar sample size' column that previously read " 499 " should read " 196 ";

- the text in 'Reference sample size' column that previously read '398" should read " 183 ";

- the text in the 'P of meta-analysis' column that previously read " 0.22 " should read " 0.15 ";

- the text in the 'P between sub-groups' column that previously read " 0.68 " should read " 0.43 ";

- the text in the 'ES (95\% CI)' column that previously read "1.36 $(0.84,2.23)$ " should read "1.60 $(0.85,30.1)$ ".

\section{Page 384, Fig. 3, 'FN incidence in cycle1.cohort study' section, ' $F$ biosimilars' row:}

- the text in the 'Study number' column that previously read " 5 " should read " 4 ";

- the text in the 'Biosimilar sample size' column that previously read "860" should read "557";

- the text in 'Reference sample size' column that previously read "6970" should read "6755";

- the text in the 'P of meta-analysis' column that previously read " 0.36 " should read " 0.35 ";

- the text in the 'ES (95\% CI)' column that previously read “1.20 $(0.81,1.78)$ " should read " $1.25(0.79,1.98)$ ".

A corrected version of Fig. 3 is shown below. 


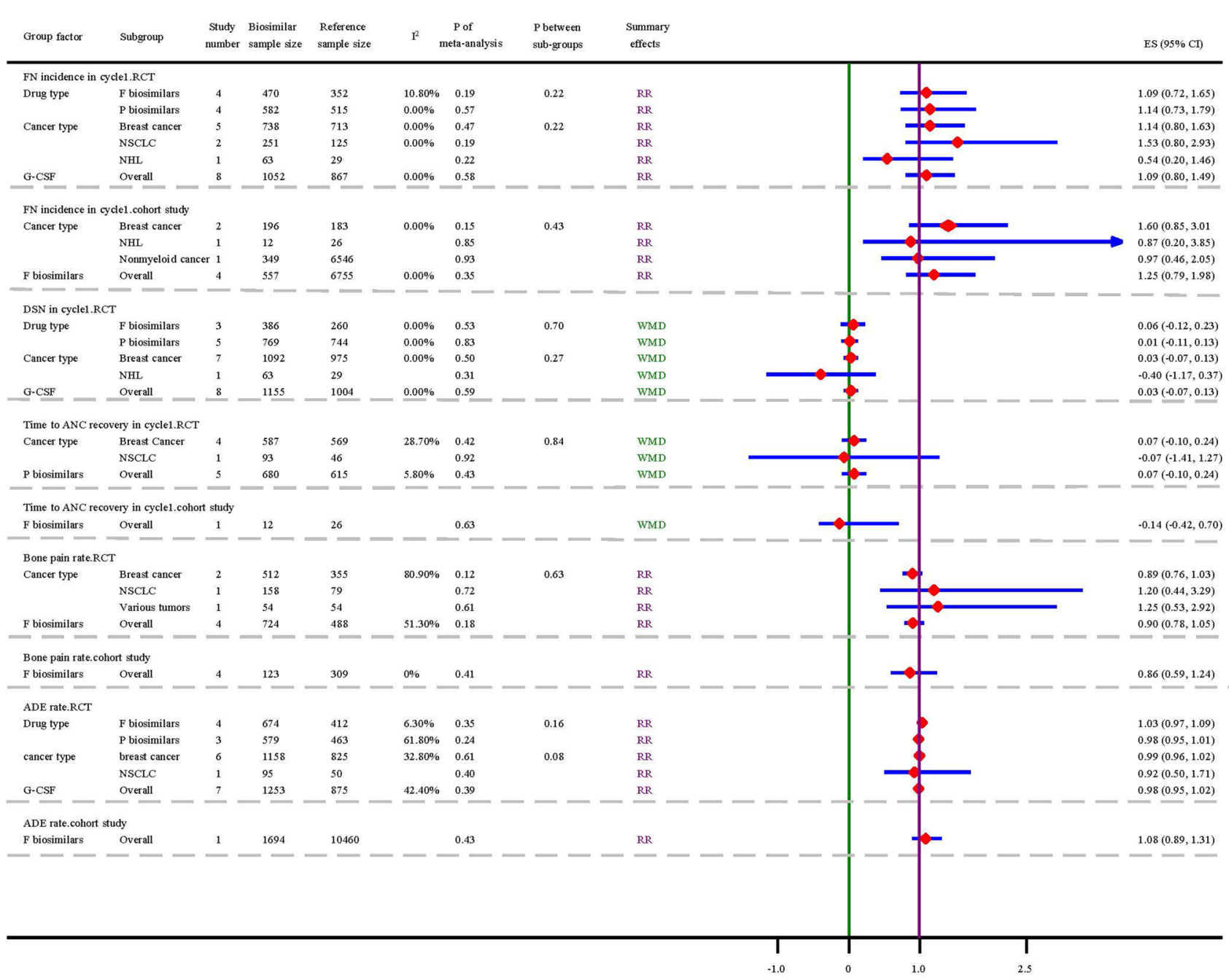

Fig. 3 Meta-analysis of G-CSF biosimilar drugs vs G-CSF drugs. $A D E$ at least one adverse drug event, $A N C$ absolute neutrophil count, $C I$ confidence interval, $D S N$ duration of severe (grade 4) neutropenia, $E S$ effect size, $F$ biosimilars filgrastim biosimilars, $F N$ febrile

Page 388, Reference \# 45: This reference should be deleted.

Electronic Supplementary Material, Supplementary Table 3, 'Puertolas et al. 2016 [25]' row: this row should be deleted.

\section{Electronic Supplementary Material, Supplementary} Table 5, ' $\mathrm{FN}$ incidence in cycle1' row:

the text in column 'study' that previously read " $5[20,21$, $25,27,28]$ " should read " 4 [20, 21, 27, 28]" neutropenia, $G-C S F$ granulocyte colony-stimulating factors, $N H L$ non-Hodgkin's lymphoma, NSCLC nonsquamous non-small-cell lung cancer, $P$ biosimilars pegfilgrastim biosimilars, $R C T$ randomized controlled trial, $R R$ risk ratio, $W M D$ weighted mean differences

the text in column 'patient' that previously read "7830" should read "7312"

Electronic Supplementary Material, page 9, reference \#25: this reference should be deleted 